\title{
Design and Analysis of Automobiles Manufacturing System Based on Simulation Model
}

\author{
Razman Bin Mat Tahar \& Ali Asghar J.Adham \\ Faculty of Technology Management, University Malaysia Pahang, Kuantan 26300, Pahang, Malaysia \\ E-mail: aliadham12@yahoo.com
}

\begin{abstract}
The global financial crisis, brewing for a while, really started to show its in automobiles Automotive manufacturing is complex and includes the coordination of design and manufacturing. In the manufacturing approaches over the past few years, assembly has been a key issue and it seems simulation models are usually tailored to address a narrow set of industrial issues. This paper describes the development of manufacturing system design, operation, and maintenance based on Simulation. The model is being developed at two different levels: the supply chain, the assembly plant. A solution that may optimize one performance measure may deteriorate other performance solution difficult. The resulting algorithms are comparable to the simulation in terms of success rate, assembly times, peak forces and moments, and have assembly times superior to those of a benchmark blind search algorithm.
\end{abstract}

Keywords: Manufacturing assembly, Automotive assembly, Supply chain manufacturing, Simulation manufacturing, Simulation industry

\section{Introduction}

Manufacturing simulation is one of the most powerful analysis tools available to those responsible for the design and operation of manufacturing systems and the model can be reconfigured for many situations in automotive assembly(A. Dalvi; M. Guay. 2009). Manufacturing application include both facility design, as well as enterprise-wide supply chain modeling. However, typical manufacturing model is usually used either to predict system performance or to compare two or more systems design or scenarios(Scott Miller. 2000).

In the main, Generic simulation identifies common model input and output data interfaces that could be standardized for particular modeling level and simulation case studies. Simulation study is essentially a demanding and resource intensive task involving at least two major activities: Model building and experimentation (Benny Tjahjono; Raúl Fernández. 2008). In an increasingly competitive world, simulation has become a very powerful tool for the planning, design, and control of manufacturing systems to facilitate current and future training, experimentation, and testing of interoperability of software(Poornachandra Rao, Vira Chankong. 2005). Accordingly, many companies had applied simulation to the decision making processes.

As pointed out by Pegden, because of fierce competition, industry is now being forced into implementing expensive factory automation and is, therefore, carefully reexamining it. Operating policies and procedures. Typical manufacturing competitive priorities or strategies are low cost, delivery, flexibility and quality (Fritz J; Dolores H. Russ. 1997).

Vehicles successively pass through three different shops (body, painting and assembly). Each of these shops has specific constraints and perturbations that locally modify the pre-defined sequence of vehicles(Jitesh Kapadia. 1998). The assembly lines consist of a set of stations that produce one or many products. Each product must go through each station in the same order. In order for an assembly line to function properly, the operations in the stations must be balanced with respect to cycle time (Mehrdad Zoroufi. 2004). The analysis evaluated the cost structure for conventional vehicle manufacturing and retailing and assigned shares of the manufacturer's suggested retail price to various cost contributors (Deogratias Kibira; Charles R. McLean. 2007).

This paper focus on development of Vehicle manufacturing system design, operation, and maintenance based on Simulation. The process involves a number of operations, which require assembling together accounts of fabricated and purchased components, subassemblies, and systems. In addition, the overall goal to virtual manufacturing environment process to provide interoperability tests support to the manufacturers, researchers, and standards organizations will be achieve by using ARENA software for simulation model as shown in figurel. 


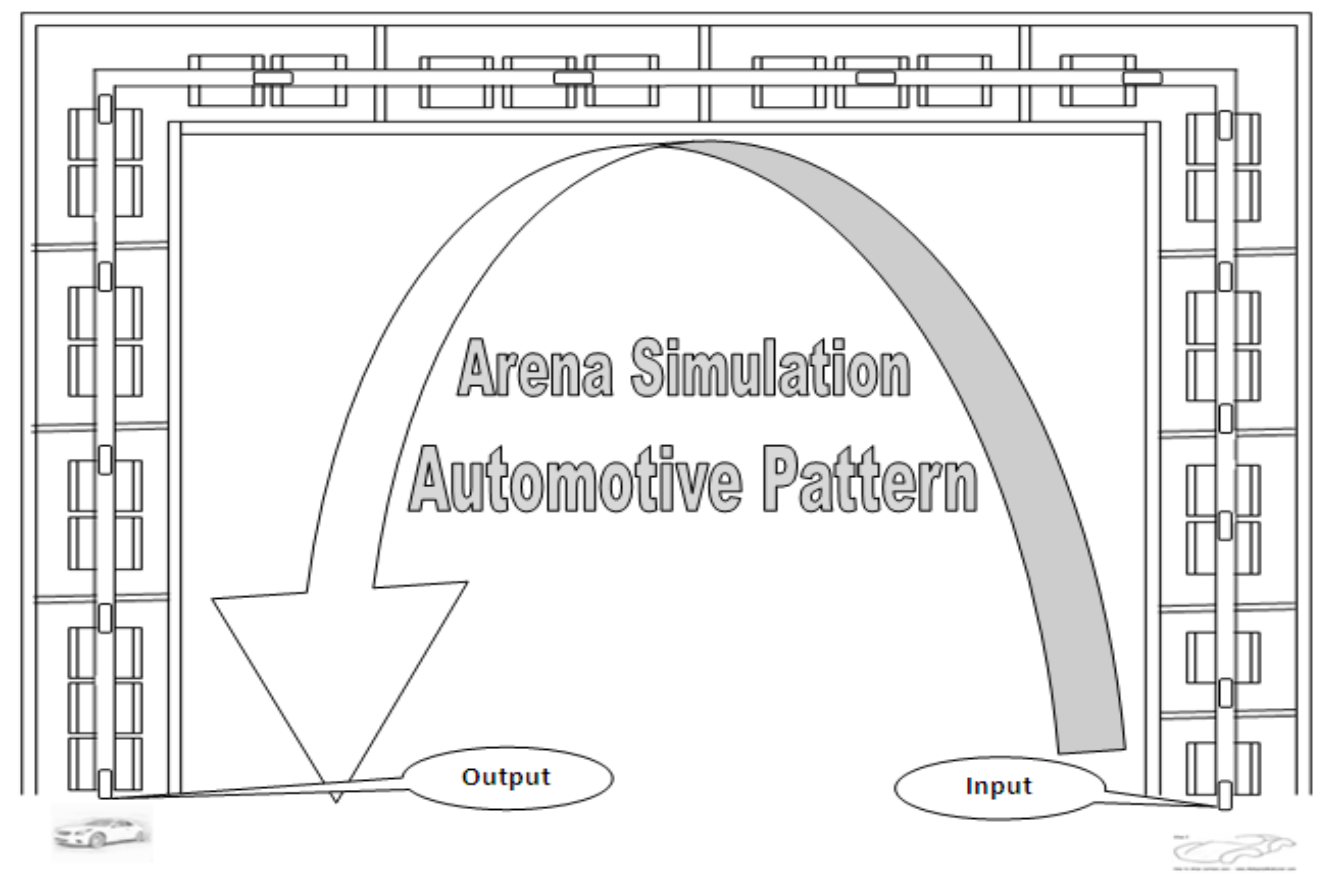

Fig. (1) The product stage Using ARENA Software

The sequence of the work presented here are:

- Identify facilities, systems, operations, parts, and processes in automotive manufacturing assembly to develop the model.

- Develop the simulation model of the final assembly plant.

- Perform conformance and acceptance testing using simulations to create the specified range of inputs for a delivered system or process.

- Evaluate effectiveness of new interface standards and protocols to meet manufacturing industry needs.

Finally, the expected result shows that the company could explore new operating procedures, organizational structures, etc. without disrupting ongoing operations in addition to controlling the time in term of compressed or expanded, allowing the speeding or slowing down of product process in order to achieve the target.

\section{Theoretical background}

\subsection{Manufacturing strategy}

Manufacturing strategy has been defined as the pattern of decisions that, over time, enables a business unit to achieve a desired manufacturing structure, infrastructure and set of specific capabilities.

Many older vehicles had separate underlying stiffening structures and bodies for housing the passengers. Therefore, the design requires more materials and results in a heavier car and contributes to raise the costs. Generally, the automotive production process consists of three major sections: the body shop, the paint shop, and the trim assembly shop. In addition, to other sections which are the power train assembly (consisting of the engine, gearbox, clutch, and transmission), and the press shop if body parts are stamped at the plant. Also, there is a final testing process where vehicles are checked for water tightness and a stationary road test (Deogratias Kibira; Charles R. McLean. 2007).

At assembly shop, some parts are usually assembled into the body before it is merged with the power system; the process is called "body drop" since it is the body that is usually lowered onto the chassis/power system. Typically, the most trim assembly operations are carried out manually and it needs a worker or two on either side of the line at a station. On the other hand, there is sufficient space on which to work, equipment and tools specific to the station as well as racks or bins on which the parts are stored for assembly.

An assembly line is a special case of the product layout. Adopting a product layout makes sense when the batch size of a given product or part is large relative to the number of different products or parts produced. Assembly 
lines refer to progressive assembly operations linked by some material handling device. Virtually any product that has multiple parts and is produced in large volume is produced on assembly lines to some degree (Jitesh Kapadia. 1998).

A variety of configuration options can be made by the customer. The options on the car are that it can be either two or four door. A two-door car will require a different body side panel from a four-door car. Today most cars are manufactured according to the customer requirement (Deogratias Kibira; Charles R. McLean. 2007).

\subsection{Simulation Model}

Since the simulation is heavily based upon computer science, mathematics, probability theory and statistics: yet the process of simulation modeling and experimentation remains very much an intuitive art. Simulation is a very general and somewhat ill-defined subject. For the purpose of this paper, we will define simulation as, "the process of designing a computerized model of a system (or process) and conducting experiments with this model for the purpose either of understanding the behavior of the system and/or of evaluating various strategies for the operation of the system." Thus we will understand the process of simulation to include both the construction of the model and the analytical use of the model for studying a problem.

The simulation runs in a computer workstation for it costs a lot of computing resources. The simulation process is: Firstly, design variables is assigned with initial values and then product analysis is executed with ARENA mode; secondly, design variables are extracted from Matlab software.

\section{The Proposed Method}

Manufacturing simulation has been one of the primary application areas of simulation technology. It has been widely used to improve and validate the design of a wide range of manufacturing system (Jonathan J.Shi. 2000). Hence, Computer simulation is a powerful tool for analyzing and optimizing real-world system with a wide range of successful application (Michael W.Baenet. 2000). In this study, the computer simulation by using ARENA software will be used to improve the production line through supply chain and assemble.

3.1 Supply chains are large system consisting of many entities interacting in complex ways. The challenge faced by companies is how to design and manage such system (Angelisa Elisabeth. 2003). The simple pattern of supply chain is shows in figure 2.

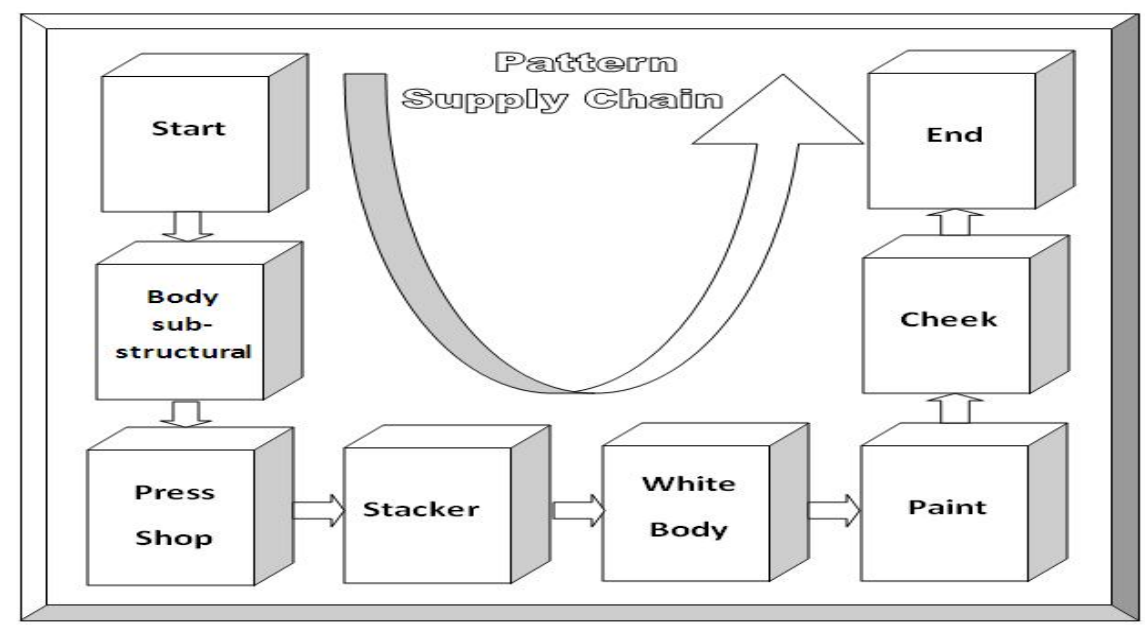

Fig. (2) Pattern for Supply Chain

3.2 Assembly of operations with production of parts. From a practical stand point of view it is preferred to have a system design which has a mix of operations and integration efficiencies, compared to a design which outperforms on normal criteria and completely to be distinguished by integration of assembly operations with production of parts. Figure 3 shows the simple pattern for assembly. 


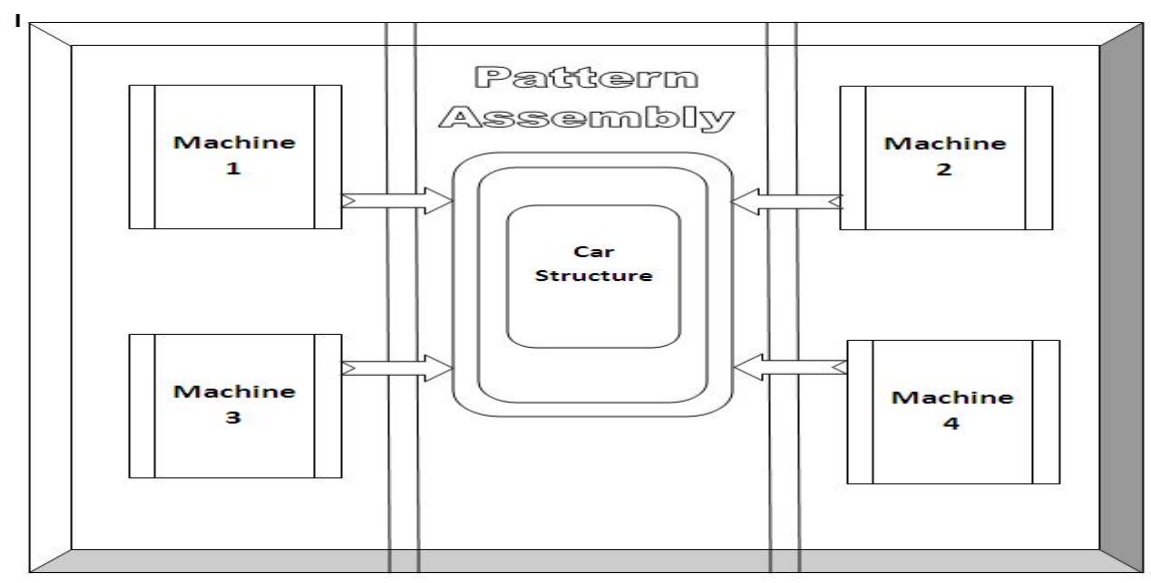

Fig. (3) Pattern for Assembly

\section{Simulation Result}

The using of simulation model is to estimate objective function values when they are needed during the course of optimization, and uses a new rule for accepting a candidate solution that depends on the individual estimated objective function values.

The automotive manufacturing was applied according to the ARENA software design. The estimated operation time was one month for 24 hours daily. In this month, 4518 cars will be produced as shown in table 1 . The table reviews all information about the software. Thus, simulation modeling can be considering as an experimental and applied methodology that seeks to accomplish the use of model to predict future behavior.

The model results are shown bellow to converge rapidly on an inventory management problem.

Replication ended at time: 720 Hours

Statistics were cleared at time: 1.0 Hours

Statistics accumulated for time: 719.0 Hours

Base Time units: Hours

Simulations run time: 2.37 minutes

Table 1. Result application to simulation model

\begin{tabular}{|l|l|l|l|l|l|}
\hline Identifier & Average & Half Width & Min & Max & Observations \\
\hline Production time & 4.67 counters & Corr & 4.66 & 4.67 & 4518 \\
\hline Identifier & & Count & Limit & & \\
\hline Number Produced & 4518 Infinite & \multicolumn{5}{ll}{} \\
\cline { 1 - 3 } & &
\end{tabular}

To clarify the results of applying the model, it can be observe from the table the difference between the production time and the amount of products, as well as the difference between the production times in the normal case where the proposed model is not used. The different in production time before and after using AREANA software is show in figure 4. 


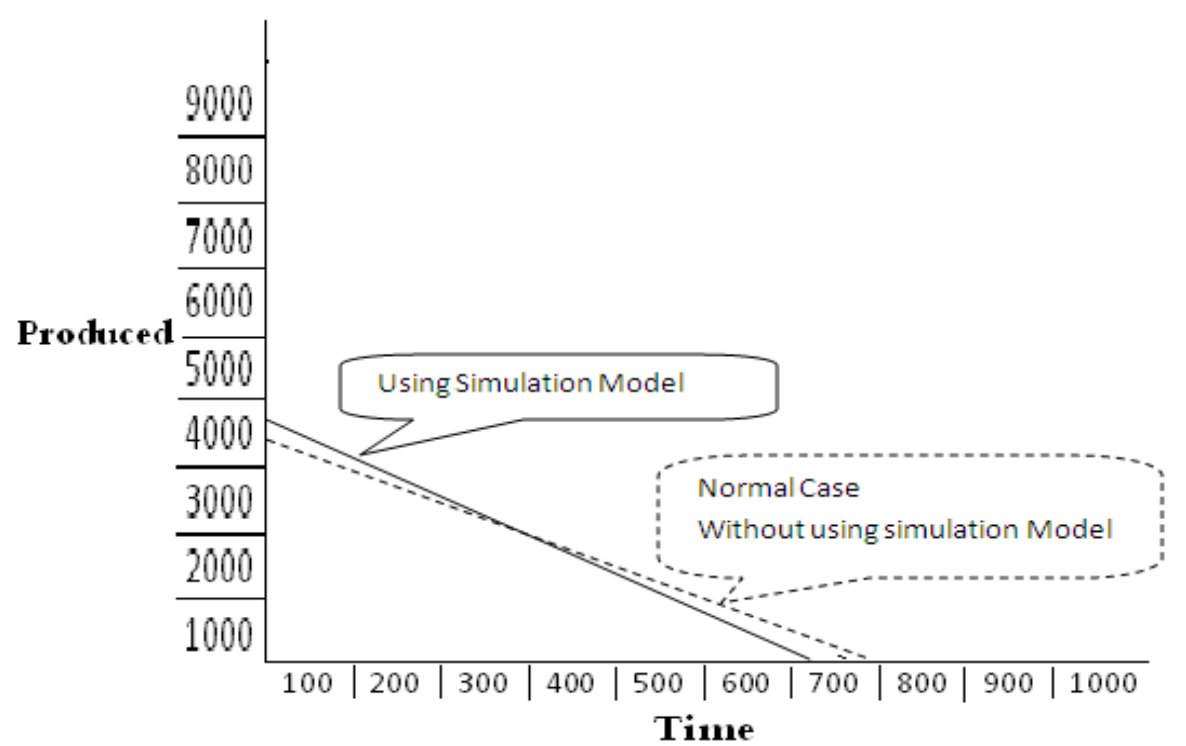

\section{Conclusions}

Fig. (4) Chart for deferent time before and after using simulation model

The model has been integrated with the supply chain simulation developed in another system by using (ARENA).The main focus of this paper was to simulate a production facility that manufactured car based on simulation model. The amount of products and the time saving showed the difference between before and after using simulation. The obtained data from the model can be applied to select the line produce to achieve optimum product. Finally, by introducing the model the company could explore new policies, operating procedures, and organizational structures without disrupting ongoing operations.

\section{References}

A. Dalvi; M. Guay. (2009). Control and Real-Time Optimization Of An Automotive Hybrid Fuel Cell Power System. 2007, Control Engineering Practice. 17 (2009) 924-938.

Angelisa Elisabeth. (2003). The Relationships Among Supply Chain Characteristics Logistics and Manufacturing Strategies, and Performance. 2003, thesis of Ohio University.

Benny Tjahjono; Raúl Fernández. (2008). Practical Approach To Experimentation In A Simulation Study. Proceedings of the 2008 Winter Simulation Conference.

Deogratias Kibira; Charles R. McLean. (2007). Generic Simulation Of Automotive Assembly For Interoperability Testing.2007, M.D. 20899, U.S.A, Simulation Conference.

Fritz J; Dolores H. Russ. (1997). Simulation Of Two Manufacturing Systems For A Car Manufacturing Company. 1997, thesis College of Engineering and Technology Ohio University.

Jitesh Kapadia. (1998). Maintaining Optimum Balance Between Multiskilling And Inventory In Assembly Line Operations Under Dynamic Demand. 1998, Proceedings of DETC'98 ASME Design Engineering Technical Conferences

Jonathan J.Shi. (2000). "Object-Oriented Technology for enhancing activity-based modeling functionality", Simulation conferences.

Mehrdad Zoroufi. (2004). Manufacturing Process Effects On Fatigue Design And Optimization Of Automotive Components- An Analytical And Experimental Study. 2004, The University of Toledo.

Michael W.Baenet. (2000). Charles J.Miller. "Analysis the Virtual Enterprise Using Distributed Supply chain Modeling and Simulation: An application of e-scor". 2000, simulation conference.

Poornachandra Rao, Vira Chankong. (2005). "Design of cellular manufacturing systems with assembly considerations “,2005,ScienceDirect.

Scott Miller. (2000). "Introduction to Manufacturing simulation”, 2000, simulation conference. 